\title{
Three PT-symmetric Hamiltonians with completely different spectra
}

\author{
Francisco M. Fernández ${ }^{1}$ and Javier Garcia \\ INIFTA (UNLP, CCT La Plata-CONICET), División Química Teórica, Blvd. 113 \\ S/N, Sucursal 4, Casilla de Correo 16, 1900 La Plata, Argentina
}

\begin{abstract}
We discuss three Hamiltonians, each with a central-field part $H_{0}$ and a PT-symmetric perturbation $i g z$. When $H_{0}$ is the isotropic Harmonic oscillator the spectrum is real for all $g$ because $H$ is isospectral to $H_{0}+g^{2} / 2$. When $H_{0}$ is the Hydrogen atom then infinitely many eigenvalues are complex for all $g$. If the potential in $H_{0}$ is linear in the radial variable $r$ then the spectrum of $H$ exhibits real eigenvalues for $0<g<g_{c}$ and a PT phase transition at $g_{c}$.
\end{abstract}

Key words: PT-symmetry, central-field part, Stark effect, PT phase transition, broken PT symmetry

\section{Introduction}

It is known since long ago that some non-Hermitian operators may exhibit real eigenvalues [1, 2]. This fact remained a somewhat exotic mathematical subject till Bender and Boettcher [3] suggested that those operators may exhibit unbroken parity-time (PT) symmetry. From then on the problem quickly developed into a prolific field of research [4] (and references therein).

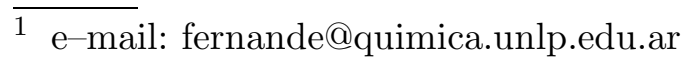


In a roughly general way we may say that most of the studied problems are represented by Hamiltonian operators of the form $H=H_{0}+\lambda H^{\prime}$, where $H_{0}$ is parity-invariant $P H_{0} P=H_{0}$ and $H^{\prime}$ is parity antisymmetric $P H^{\prime} P=-H^{\prime}$, where $P$ is the parity operator. If $\lambda=i g$ is imaginary (where $g$ is obviously real) then $H$ is PT symmetric: $P T H P T=H$, where $T$ is the time-reversal operator [5].

In the beginning, most of the models studied were mainly one-dimensional [3, 4, 6, 7, in which case $H_{0}$ only exhibits parity symmetry and its eigenfunctions $\psi^{(0)}$ are even or odd: $P \psi^{(0)}= \pm \psi^{(0)}$ but later the researchers began to look for multidimensional examples [8 15]. It was suggested that space-time (ST) symmetry could be a suitable generalization of the PT one [16]. In this case $S H_{0} S=H_{0}$ and $S H^{\prime} S=-H^{\prime}$, where $S$ is a unitary operator such that $S^{\dagger}=S^{-1}=S$. Most of the effort was devoted to find new multidimensional non-Hermitian Hamiltonians with real spectra.

In the multidimensional case $H_{0}$ and $H$ may exhibit more complex symmetry that is conveniently described by means of group theory. In this way Fernández and Garcia [17, 18] and Amore et al [19, 20] found that some ST-symmetric Hamiltonians exhibit broken ST symmetry for all values of $g$. The main conjecture was that ST symmetry may be unbroken for some values of $g$ provided that $S$ is the only member of a class in the point group for $H_{0}$ [20]. This appeared to be the case when $S=P$.

The purpose of this paper is the discussion of three PT-symmetric Hamiltonians for which $H_{0}=p^{2} / 2+V(r)$ exhibits central-field symmetry and $H^{\prime}=z$. The resulting Hamiltonian $H$ exhibits cylindrical symmetry and may be viewed as a kind of Stark effect with imaginary electric field. In Section 2 we outline the main ideas of PT symmetry as well as a simple argument based on perturbation theory [17 20]. In Section 3 we briefly discuss the general case. In sections 4, 5] and 6] we show that the models with $V(r)=r^{2} / 2, V(r)=-1 / r$, 
and $V(r)=r$, respectively, exhibit completely different spectra. Finally, in Section 7 we summarize the main results of the paper and draw conclusions.

\section{$2 \quad$ Parity-time symmetry}

Let $A=P T=A^{-1}$ be the antiunitary operator given by the product of the parity $P$ and time-reversal $T$ operators [3,5]. The Hamiltonian operator $H$ is said to be $P T$ symmetric if

$$
A H A^{-1}=H \text {. }
$$

If

$$
H \psi=E \psi,
$$

then

$$
A H \psi=A H A^{-1} A \psi=H A \psi=A E \psi=E^{*} A \psi .
$$

If $A \psi=a \psi, a$ being a complex number, then we say that PT symmetry is unbroken and $E=E^{*}$. It is not difficult to prove that $|a|=1$. Fernández and Garcia [21] found a case in which $A \psi \neq a \psi$ and still $E$ is real. They proposed the supposedly more general condition $H A \psi=E A \psi$ for the occurrence of real spectrum; that is to say, when $\psi$ and $A \psi$ are two linearly independent eigenfunctions of $H$ with the same eigenvalue $E$. This situation does not take place unless the spectrum of $H$ is degenerate. However, further analysis reveals that both conditions are equivalent. In fact, if we choose $\varphi=c_{1} \psi+c_{2} A \psi$, where $c_{2}^{*}=a c_{1}$ and $c_{1}^{*}=a c_{2}$, then $A \varphi=a \varphi$. It is worth adding that none of these conditions is of practical utility to predict whether $H$ will have real eigenvalues or not because one commonly ignores the effect of $A$ on the eigenvectors of $H$. 
Most of the examples studied so far are of the form

$$
H=H_{0}+\lambda H^{\prime}
$$

where

$$
P H_{0} P=H_{0}, T H_{0} T=H_{0}, P H^{\prime} P=-H^{\prime}, T H^{\prime} T=H^{\prime}
$$

and $\lambda=i g$, where $g$ is real. Since $T \lambda H^{\prime} T=\lambda^{*} T H^{\prime} T=-\lambda H^{\prime}$ then $A H A=H$. Some useful information on the spectrum of $H$ is given by the perturbation series

$$
E=\sum_{j=0} E^{(j)} \lambda^{j}
$$

because if at least one coefficient of odd order $E^{(2 i+1)}$ is nonzero then $E$ is expected to be complex for sufficiently small $g$. In such a case the PTphase transition [14] takes place at the trivial Hermitian limit $g=0$. If we write $H(\lambda) \psi_{m}=E_{m}(\lambda) \psi_{m}$ then $P H(\lambda) \psi_{m}=P H(\lambda) P P \psi_{m}=H(-\lambda) P \psi_{m}=$ $E_{m}(\lambda) P \psi_{m}$. If $\psi_{m}$ and $P \psi_{m}$ are linearly dependent, then $E_{m}(-\lambda)=E_{m}(\lambda)$ and all the perturbation corrections of odd order vanish; otherwise $P \psi_{m}=\psi_{n}$, $E_{m}(-\lambda)=E_{n}(\lambda)$ and we cannot draw a conclusion so easily. The latter case may only take place when the spectrum of $H$ is degenerate. In many cases it suffices to calculate the simplest, straightforward perturbation correction of first order $E^{(1)}$ [17 $[20$.

\section{Stark effect}

Consider the Hamiltonian operator

$$
H=-\frac{1}{2} \nabla^{2}+V(r)+\lambda z,
$$


where $V(r)$ is spherically symmetric (depends only on $r$ ). The eigenfunctions of $H_{0}=H(\lambda=0)$

$$
H_{0} \psi_{\nu l m}^{(0)}=E_{\nu l}^{(0)} \psi_{\nu l m}^{(0)}
$$

are also eigenfunctions of the angular momentum operators $L^{2}$ and $L_{z}$

$$
\begin{aligned}
L^{2} \psi_{\nu l m}^{(0)} & =l(l+1) \psi_{\nu l m}^{(0)} \\
L_{z} \psi_{\nu l m}^{(0)} & =m \psi_{\nu l m}^{(0)}, \\
l & =0,1, \ldots, m=0, \pm 1, \ldots, \pm l
\end{aligned}
$$

In spherical coordinates the eigenfunctions can be factored as

$$
\psi_{\nu l m}^{(0)}(r, \theta, \phi)=R_{\nu l}(r) Y_{l}^{m}(\theta, \phi),
$$

where $R_{\nu l}(r)$ is the radial part, $\nu=0,1, \ldots$, is the radial quantum number and $Y_{l}^{m}(\theta, \phi)$ are the spherical harmonics. Since the eigenvalues of $H_{0}$ do not depend on $m$ they are at least $(2 l+1)$-fold degenerate.

The perturbation $H^{\prime}=z$ breaks the degeneracy of the spectrum of $H_{0}$ but the states with $m>0$ remain two-fold degenerate because the eigenvalues of $H$ do not depend on the sign of the magnetic quantum number $m$.

Since

$$
P \psi_{\nu l m}^{(0)}=(-1)^{l} \psi_{\nu l m}^{(0)}
$$

and $P z P=-z$ the matrix elements

$$
z_{\nu l m}^{\nu^{\prime} l^{\prime} m}=\left\langle\psi_{\nu l m}^{(0)}|z| \psi_{\nu^{\prime} l^{\prime} m}^{(0)}\right\rangle
$$

are zero when $l-l^{\prime}$ is even. The perturbation corrections of first order to the energy $E_{\nu l m}^{(1)}$ are given by the eigenvalues of the matrix with elements $z_{\nu l m}^{\nu^{\prime} l^{\prime} m}$. We will discuss three examples in the subsequent sections. 


\section{Isotropic harmonic oscillator}

When

$$
V(r)=\frac{1}{2} r^{2}
$$

the Schrödinger equation is exactly solvable and the eigenfunctions and eigenvalues are given by

$$
\begin{aligned}
\psi_{n_{1} n_{2} n_{3}}(x, y, z) & =\varphi_{n_{1}}(x) \varphi_{n_{2}}(y) \varphi_{n_{3}}(z+\lambda), \\
E_{k} & =\left(k+\frac{3}{2}\right)-\frac{1}{2} \lambda^{2}, k=n_{1}+n_{2}+n_{3}, \\
n_{1}, n_{2}, n_{3} & =0,1, \ldots,
\end{aligned}
$$

where $\varphi_{n}(q)$ is an eigenfunction of the one-dimensional harmonic oscillator $H_{H O}=-\frac{1}{2} \frac{d^{2}}{d q^{2}}+\frac{1}{2} q^{2}$.

Since

$$
\begin{aligned}
A \psi_{n_{1} n_{2} n_{3}}(x, y, z) & =\psi_{n_{1} n_{2} n_{3}}(-x,-y,-z)^{*}=\varphi_{n_{1}}(-x) \varphi_{n_{2}}(-y) \varphi_{n_{3}}\left(-z+\lambda^{*}\right) \\
& =(-1)^{k} \psi_{n_{1} n_{2} n_{3}}(x, y, z)
\end{aligned}
$$

then the PT symmetry is unbroken for all $g$ which accounts for the fact that the eigenvalues in equation (14) are real for all $g$.

Although in this case the approximate analysis based on perturbation theory may appear to be unnecessary we carry it out anyway merely for comparison purposes. To begin with, note that $P \psi_{n_{1} n_{2} n_{3}}^{(0)}(x, y, z)=(-1)^{k} \psi_{n_{1} n_{2} n_{3}}^{(0)}(x, y, z)$. The perturbation correction of first order to a given energy level $E_{k}^{(0)}$ is given by matrix elements of the form

$$
z_{n_{1} n_{2} n_{3}}^{m_{1} m_{2} m_{3}}=\left\langle\psi_{n_{1} n_{2} n_{3}}^{(0)}|z| \psi_{m_{1} m_{2} m_{3}}^{(0)}\right\rangle
$$


that vanish for all degenerate states because $k=n_{1}+n_{2}+n_{3}=m_{1}+m_{2}+m_{3}$. Therefore, $E_{k}^{(1)}=0$ for all the states of the PT Stark effect in the isotropic harmonic oscillator. This result is consistent with the form of the exact eigenvalues (14) that depend on $g^{2}$.

There is another way to prove that the PT symmetry for this problem remains unbroken for all values of $g$. The proof is based on the fact that $H$ can be written in terms of a similarity transformation of $H_{0}$ :

$$
H=U H_{0} U^{-1}+\frac{g^{2}}{2}, U=e^{-g p_{z}}
$$

where $p_{z}=-i \frac{d}{d z}$. Obviously, $H_{0}$ and $U H_{0} U^{-1}$ are isospectral [22].

\section{Hydrogen atom}

The unperturbed eigenvalues for the Coulomb interaction

$$
V(r)=-\frac{1}{r}
$$

are given by

$$
E_{n}^{(0)}=-\frac{1}{2 n^{2}}, n=\nu+l+1
$$

Therefore, there are pairs of degenerate states $\psi_{\nu l m}^{(0)}, \psi_{\nu^{\prime} l^{\prime} m}^{(0)}$ for which $l-l^{\prime}=$ $\nu^{\prime}-\nu$ is odd and the corresponding matrix elements $z_{\nu l m}^{\nu^{\prime} l^{\prime} m}(12)$ are nonzero. In such cases, which for real $\lambda$ give rise to what is commonly known as linear Stark effect [23, 24], the perturbation correction of first order is nonzero and the eigenvalues of $H$ are complex for $g \neq 0$.

The Schrödinger equation for this problem is separable in parabolic coordinates and the exact calculation of the perturbation corrections in terms of the 
parabolic quantum numbers $n_{1}=0,1, \ldots, n_{2}=0,1, \ldots$ and $m=0, \pm 1, \ldots$ is straightforward [25]. It is customary to write the perturbation series

$$
E_{n q|m|}=\sum_{j=0}^{\infty} E_{n q|m|}^{(j)} \lambda^{j}
$$

in terms of the quantum numbers $n=n_{1}+n_{2}+|m|+1$ and $q=n_{1}-n_{2}$ [25]. All the coefficients of odd order vanish when $q=0$ but the states with $q \neq 0$ are expected to be complex when $g \neq 0$.

The argument based on perturbation theory just outlined is sufficient to conclude that this model exhibits complex eigenvalues when $g \neq 0$ and that the PT phase transition [14] takes place at the trivial Hermitian limit $g=0$. Nevertheless, we will show some numerical results to illustrate the point. Here we choose the most efficient method of Benassi and Grecchi [26] that is based on the separation of the Schrödinger equation in squared-parabolic coordinates. Since the details of this approach have been given elsewhere [26 28], here we just show the results. Figure1 shows the real and imaginary parts of the lowest eigenvalues. It is clear that the PT phase transition takes place at the trivial Hermitian limit as already argued above.

The remarkable difference between the spectra of this problem and the previous one can be traced back to the symmetry of $H_{0}$. The general central-field model is invariant under the group $\mathrm{O}(3)$ while, on the other hand, the hydrogen atom is invariant under the group $\mathrm{O}(4)$ [29]. Such higher symmetry is due to the conservation of the Runge-Lenz vector in the latter model. Thus, the higher symmetry of $H_{0}$ appears to be the reason why the PT symmetry is broken for all $g$ in the perturbed hydrogen atom. While the $k$-th harmonicoscillator eigenvalue $E_{k}^{(0)}$ is $\frac{(k+1)(k+2)}{2}$-fold degenerate, the $n$-th eigenvalue of the hydrogen atom $E_{n}^{(0)}$ is $n^{2}$-fold degenerate. The greater degeneracy of the latter model allows the appearance of nonzero matrix elements $z_{\nu l m}^{\nu^{\prime} l^{\prime} m}$ and nonzero perturbation corrections of first order. 


\section{Linear potential}

As a nontrivial example we consider the linear potential

$$
V(r)=r
$$

In this case we cannot solve the eigenvalue equation for $H_{0}$ exactly but we can nevertheless calculate the perturbation correction of first order to any energy level $E_{\nu l}^{(0)}$ because it is determined by matrix elements of the form

$$
z_{\nu l m}^{\nu l m^{\prime}}=\left\langle\psi_{\nu l m}^{(0)}|z| \psi_{\nu l m^{\prime}}^{(0)}\right\rangle
$$

which vanish for all sets of quantum numbers as argued in Section 3. Therefore, $E_{\nu l|m|}^{(1)}=0$ and there is a chance that PT symmetry may be unbroken for sufficiently small $g$.

We can calculate approximate eigenvalues by means of diagonalization of a suitable matrix representation of the Hamiltonian. For simplicity, here we choose the nonorthogonal Slater-type basis set

$$
B=\left\{r^{n} e^{-\alpha r} Y_{l}^{m}(\theta, \phi), n, l,|m|=0,1, \ldots\right\}
$$

Present numerical results show that this problem exhibits the usual spectral pattern common to most PT-symmetric Hamiltonians studied by other authors; that is to say, unbroken PT symmetry for $0<g<g_{c}$. For sufficiently small values of $g$ the eigenvalues are real. As $g$ increases two eigenvalues approach each other, coalesce at an exceptional point [30 33] $g_{i} \geq g_{c}$ becoming a pair of complex conjugate numbers for $g>g_{i}$. This behaviour is illustrated by figures 2 , 3 and 4, for $m=0,1,2$, respectively. Those results were obtained by diagonalization of the matrix representation of the Hamiltonian operator in the Slater basis set (23) with $\alpha=2$. The irregular lines reflect errors in the calculation of the eigenvalues originated in the quasi linear dependence of the 
basis set. This shortcoming of the present approach becomes more noticeable as the number of radial basis functions increases. Although our numerical results are not extremely accurate and are restricted to the lowest eigenvalues for the reason just indicated, they appear to suggest that the smallest exceptional point $g_{c}$ may be nonzero and that there is a PT phase transition at such point. We think that a more accurate calculation is not necessary to illustrate the difference between this model and the other two ones discussed above.

\section{Conclusions}

In this paper we have discussed three Hamiltonians given by three different central-field Hermitian parts and the same non-Hermitian PT-symmetric perturbation. Although at first sight they appear to be similar, they exhibit completely different spectra. In the case of the isotropic harmonic oscillator the PT symmetry is unbroken and the spectrum is real for all $g$. The reason is that $H$ and $H_{0}$ are related by the similarity transformation (17). On the other hand, the PT symmetry is broken for all $g$ in the case of the hydrogen atom. Quite in between the linear radial potential appears to exhibit unbroken PT symmetry for all $0<g<g_{c}$ and a phase transition at some $g_{c}$ that we were unable to determine.

The remarkable difference among the spectra of such seemingly similar Hamiltonians is due to the symmetry of $H_{0}$. As a general rule the higher the symmetry of $H_{0}$ the more likely the occurrence of complex eigenvalues and the Hamiltonian for the hydrogen atom exhibits the greatest symmetry by far. We have already discussed the effect of symmetry in earlier papers [17 20] but we have not seen such a remarkable difference in the behaviour of the non-Hermitian Hamiltonians.

In closing we want to stress the fact that perturbation theory provides a useful 
hint about the nature of the spectra of a given non-Hermitian Hamiltonian. If a perturbation correction of odd order (we typically look for the first one) is nonzero then we know that the spectrum is complex for all values of $g$ (or at least for sufficiently small $g$ ). If all the available perturbation corrections of odd order are zero then there is a chance of finding real spectrum for some values of g. Obviously, this case should be investigated by more accurate calculations. As the symmetry of $H_{0}$ increases, then also increases the dimension of its eigenspaces and, consequently, the dimension of the matrix representation of the perturbation in those eigenspaces. As a result it also increases the chance of nonzero perturbation corrections of first order.

\section{References}

[1] E. Caliceti, S. Graffi, and M. Maioli, Perturbation theory of odd anharmonic oscillators, Commun. Math. Phys. 75 (1980) 51-66.

[2] G. Alvarez, Bender-Wu branch points in the cubic oscillator, J. Phys. A 28 (1995) 4589-4598.

[3] C. M. Bender and S. Boettcher, Real Spectra in Non-Hermitian Hamiltonians Having PT Symmetry, Phys. Rev. Lett. 80 (1998) 5243-5246.

[4] C. M. Bender, Making sense of non-Hermitian Hamiltonians, Rep. Prog. Phys. 70 (2007) 947-1018.

[5] C. E. Porter, Fluctuations of quantal spectra, in: C. E. Porter (Ed.), Statistical theories of spectra: fluctuations, Vol. Academic Press Inc., New York and London, 1965.

[6] F. M. Fernández, R. Guardiola, and M. Znojil, Strong-coupling expansions for the PT-symmetric oscillators $V(x)=a(i x)+b(i x)^{2}+c(i x)^{3}$, J. Phys. A 31 (1998) 10105-10112.

[7] F. M. Fernández, R. Guardiola, J. Ros, and M. Znojil, A family of complex potentials with real spectrum, J. Phys. A 32 (1999) 3105-3116. 
[8] C. M. Bender, G. V. Dunne, P. N. Meisinger, and M. Simsek, Quantum complex Hénon-Heiles potentials, Phys. Lett. A 281 (2001) 311-316.

[9] A. Nanayakkara and C. Abayaratne, Semiclassical quantization of complex Henon-Heiles systems, Phys. Lett. A 303 (2002) 243-248.

[10] A. Nanayakkara, Real eigenspectra in non-Hermitian multidimensional Hamiltonians, Phys. Lett. A 304 (2002) 67-72.

[11] A. Nanayakkara, Comparison of quantal and classical behavior of PT-symmetric systems at avoided crossings, Phys. Lett. A 334 (2005) 144-153.

[12] H. Bíla, M. Tater, and M. Znojil, Comment on: "Comparison of quantal and classical behavior of PT-symmetric systems at avoided crossings" [Phys. Lett. A 334 (2005) 144], Phys. Lett. A 351 (2006) 452-456.

[13] Q-H Wang, Level crossings in complex two-dimensional potentials, Pramana J. Phys. 73 (2009) 315-322.

[14] C. M. Bender and D. J. Weir, PT phase transition in multidimensional quantum systems, J. Phys. A 45 (2012) 425303.

[15] C. R. Handy and D. Vrincenau, Orthogonal polynomial projection quantization: a new Hill determinant method, J. Phys. A 46 (2013) 135202.

[16] S. Klaiman and L. S. Cederbaum, Non-Hermitian Hamiltonians with space-time symmetry, Phys. Rev. A 78 (2008) 062113. See also: Erratum: Non-Hermitian Hamiltonians with space-time symmetry [Phys. Rev. A 78, 062113 (2008)], Phys. Rev. A 89 (2014) 039908(E).

[17] F. M. Fernández and J. Garcia, Non-Hermitian Hamiltonians with unitary and antiunitary symmetries, Ann. Phys. 342 (2014) 195-204. arXiv:1309.0808 [quant-ph].

[18] F. M. Fernández and J. Garcia, PT-symmetry broken by point-group symmetry, J. Math. Phys. 55 (2014) 042107. arXiv:1308.6179v2 [quant-ph].

[19] P. Amore, F. M. Fernández, and J. Garcia, Non-Hermitian oscillators with Td symmetry, Ann. Phys. 353 (2014) 238-251. arXiv:1409.2672 [quant-ph] 
[20] P. Amore, F. M. Fernández, and J. Garcia, Is space-time symmetry a suitable generalization of parity-time symmetry?, Ann. Phys. 350 (2014) 533-548. arXiv:1405.5234 [quant-ph].

[21] F. M. Fernández and J. Garcia, Critical parameters for non-hermitian Hamiltonians, Appl. Math. Commun. 247 (2014) 141-151. arXiv:1305.5164 [math-ph].

[22] F. M. Fernández, Non-Hermitian Hamiltonians and Similarity Transformations, Int. J. Theor. Phys. (2015)(in the press) arXiv:1502.02694 [quant-ph].

[23] H. A. Bethe and E. E. Salpeter, Quantum Mechanics of One- And Two-Electron Atoms, (Springer, Berlin, Götingen, Heidelberg, 1957).

[24] L. D. Landau and E. M. Lifshitz, Quantum Mechanics. Non-relativistic Theory, (Pergamon, New York, 1958).

[25] F. M. Fernández, Introduction to Perturbation Theory in Quantum Mechanics, (CRC Press, Boca Raton, 2000).

[26] L. Benassi and V. Grecchi, Resonances in the Stark effect and strongly asymptotic approximants, J. Phys. B 13 (1980) 911-930.

[27] F. M. Fernández and J. Garcia, Comment on "Stark effect in neutral hydrogen by direct integration of the Hamiltonian in parabolic coordinates", Phys. Rev. A 91 (2015) 066501. On recent calculations of resonances for the Stark effect in hydrogen. arXiv:1505.01327 [quant-ph]

[28] F. M. Fernández and J. Garcia, Highly accurate calculation of the resonances in the Stark effect in hydrogen, arXiv:1506.05797 [quant-ph].

[29] M. Lieber, O(4) Symmetry of the Hydrogen Atom and the Lamb Shfft, Phys. Rev. 174 (1968) 2037-2054.

[30] W. D. Heiss and A. L. Sannino, Avoided level crossing and exceptional points, J. Phys. A 23 (1990) 1167-1178.

[31] W. D. Heiss, Repulsion of resonance states and exceptional points, Phys. Rev. E 61 (2000) 929-932. 
[32] W. D. Heiss and H. L. Harney, The chirality of exceptional points, Eur. Phys. J. D 17 (2001) 149-151.

[33] W. D. Heiss, Exceptional points - their universal occurrence and their physical significance, Czech. J. Phys. 54 (2004) 1091-1099. 

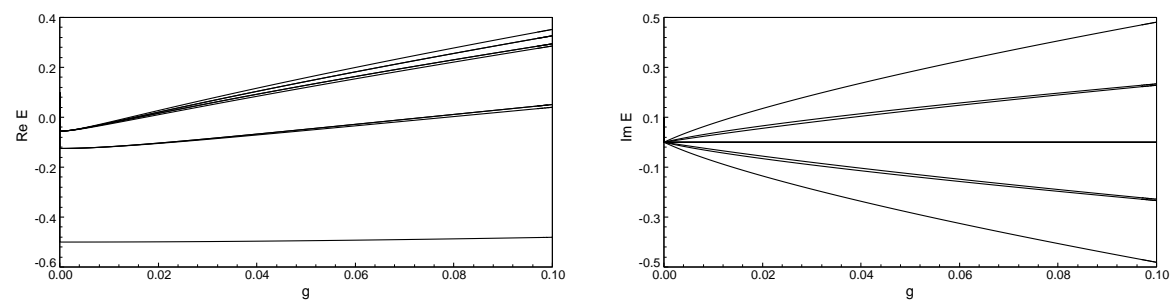

Fig. 1. Real and imaginary parts of the lowest eigenvalues of the PT-symmetric Stark effect in hydrogen
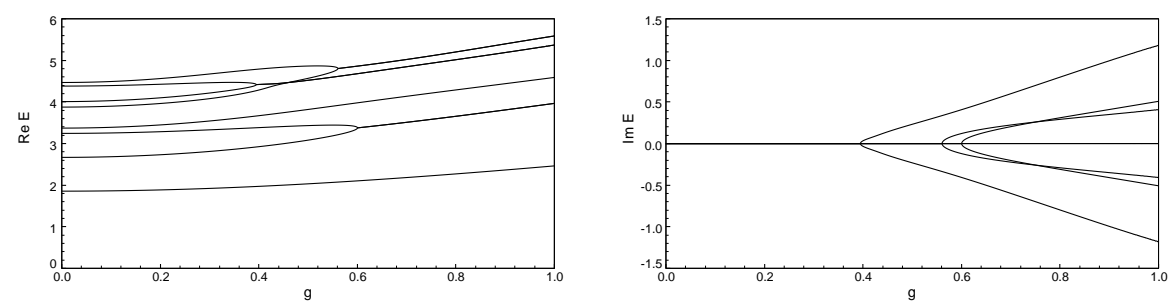

Fig. 2. Lowest eigenvalues with $m=0$ for the potential $V(r, z)=r+i g z$
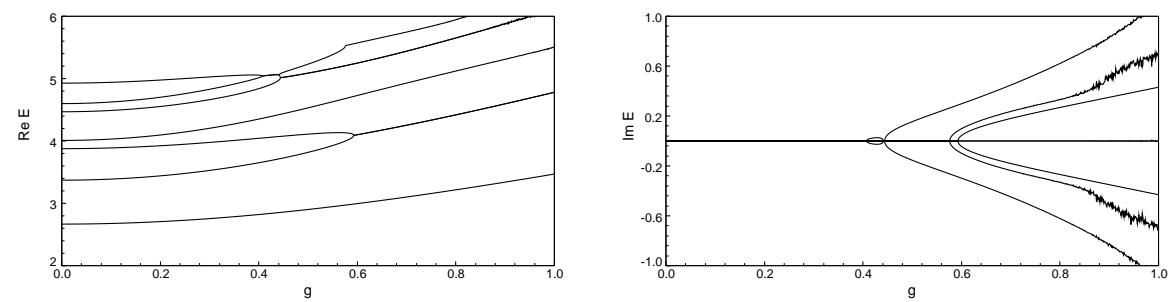

Fig. 3. Lowest eigenvalues with $m=1$ for the potential $V(r, z)=r+i g z$ with $m=1$
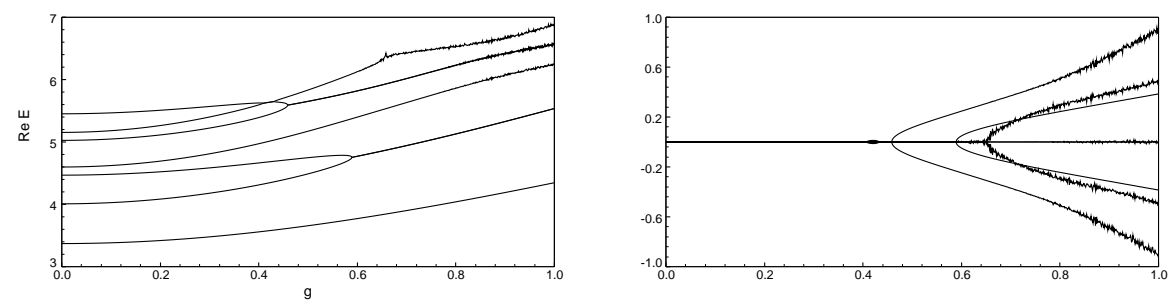

Fig. 4. Lowest eigenvalues with $m=2$ for the potential $V(r, z)=r+i g z$ 